\title{
ON A THEOREM OF HARDY AND LITTLEWOOD
}

\author{
LUIS G. BERNAL
}

(Communicated by R. Daniel Mauldin)

\begin{abstract}
In this paper, we give an extension of a classical theorem of Hardy and Littlewood on power series. Let $\varphi$ be a strictly positive function defined on some interval $(\delta, 1)$, satisfying a certain condition of limit. We prove that if $f(x)$ is the sum of a convergent power series for $0<x<1$ with nonnegative coefficients $a_{n}$ and $f(x) \sim \varphi(x)(x \rightarrow 1)$, then $S_{n} \sim \alpha \cdot \varphi\left(x_{0}^{1 / n}\right)$ $(n \rightarrow \infty)$, where $S_{n}=a_{0}+a_{1}+\cdots+a_{n}, x_{0} \in(0,1)$ and $\alpha$ depends only upon $\varphi$.
\end{abstract}

It is classical the following result about power series: Let $f(x)=\sum_{n=0}^{\infty} a_{n} x^{n}$ be a series converging for $0<x<1$, with $a_{n} \geq 0(n=0,1,2, \ldots)$. Assume that $\lim _{x \rightarrow 1}(1-x) \cdot f(x)=1$. Then $\lim _{n \rightarrow \infty}\left(S_{n} / n\right)=1$, where $S_{n}=\sum_{j=0}^{n} a_{j}$. This theorem is due to Hardy and Littlewood (see [2] and [1, pp. 481-486]). In 1930, Karamata [3] gave an elegant proof of the result, based upon the Weierstrass theorem of uniform approximation. See also [4, pp. 226-229]. In this note we use the same idea to derive an extension of the Hardy-Littlewood theorem. We substitute the function $(1-x)^{-1}$ such that $f(x) \sim(1-x)^{-1}(x \rightarrow 1)$ by a more general function $\varphi(x)$. The conclusion is similar. We shall obtain that $\lim _{n \rightarrow \infty}\left(S_{n} / \varphi\left(x_{0}^{1 / n}\right)\right)=\alpha$, where $0<x_{0}<1$ and $0 \leq \alpha<+\infty, \alpha$ depending only upon $\varphi$.

First, we state a result which we shall need in proving our theorem. Denote by $R$ the real line. A real sequence $\left\{c_{m}: m=0,1,2, \ldots\right\}$ is said to be completely monotonic if

$$
c_{m} \geq 0 \text { and }(-1)^{n} \cdot \Delta^{n} c_{m} \geq 0 \quad(m, n=0,1,2, \ldots) .
$$

Here, $\Delta^{n}(n=0,1,2, \ldots)$ denote the successive differences.

LEMMA. Let $\left\{c_{m}: m=0,1,2, \ldots\right\}$ be a real sequence. Then there is a nondecreasing function (resp. a function of bounded variation) $\sigma:[0,1] \rightarrow R$ such that $\left\{c_{m}: m=0,1,2, \ldots\right\}$ is the moment sequence for $\sigma$, i.e., $c_{m}=\int_{0}^{1} x^{m} d \sigma(x)$ $(m=0,1,2, \ldots)$, if and only if $\left\{c_{m}: m=0,1,2, \ldots\right\}$ is completely monotonic (resp. $\left\{c_{m}: m=0,1,2, \ldots\right\}$ is the difference of two completely monotonic sequences).

For the proof, see [5, pp. 100-109]. In both cases, the function $\sigma$ is unique, if we identify two functions $\sigma^{\prime}, \sigma^{\prime \prime}$ for which $\int_{0}^{1} f d \sigma^{\prime}=\int_{0}^{1} f d \sigma^{\prime \prime}$ for every continuous function $f$ on $[0,1]$. We shall say that $\sigma$ generates $\left\{c_{m}\right\}$. It is known that the set $C(h)=\{x \in(0,1): h$ is continuous at $x\}$ is conumerable in $(0,1)$, if $h$ is of bounded variation on $[0,1]$. Next, we state our result.

Received by the editors December 8, 1987.

1980 Mathematics Subject Classification (1985 Revision). Primary 40E05; Secondary 30B10, $30 \mathrm{~B} 30$.

Key words and phrases. Power series, Hardy-Littlewood theorem, Weierstrass theorem, moment sequence, completely monotonic sequence.

(C) 1988 American Mathematical Society $0002-9939 / 88 \$ 1.00+\$ .25$ per page 
THEOREM. Let $\varphi(x)$ be a strictly positive function defined on some interval $(\delta, 1)(\delta<1)$, and let $f(x)=\sum_{n=0}^{\infty} a_{n} x^{n}$ be a series converging for $0<x<1$, with $a_{n} \geq 0(n=0,1,2, \ldots)$. Assume that $f(x) \sim \varphi(x)(x \rightarrow 1)$ and that the limits $c_{m}=\lim _{x \rightarrow 1} \varphi\left(x^{m+1}\right) / \varphi(x)(m=0,1,2, \ldots)$ exist as real numbers. In addition, assume that $\left\{c_{m}: m=0,1,2, \ldots\right\}$ is the difference of two completely monotonic sequences. If $\sigma$ generates $\left\{c_{m}\right\}$ and $x_{0} \in C(\sigma)$, then

$$
\lim _{n \rightarrow \infty} \frac{S_{n}}{\varphi\left(x_{0}^{1 / n}\right)}=\alpha,
$$

where $\alpha=\int_{x_{0}}^{1} t^{-1} d \sigma(t)$.

PROOF. Consider the function $g(t)$ defined on $[0,1]$ by $g(t)=0\left(0 \leq t<x_{0}\right)$, $g(t)=1 / t\left(x_{0} \leq t \leq 1\right)$. Let $\varepsilon$ be a given positive number. Denote by $V(t)$ the total variation of $\sigma$ on $[0, t](0 \leq t \leq 1)$, and choose $\beta$ such that

$$
0<\beta<\min \left(x_{0}, 1-x_{0}\right)
$$

and

$$
\max \left(V\left(x_{0}\right)-V\left(x_{0}-\beta\right), V\left(x_{0}+\beta\right)-V\left(x_{0}\right)\right)<(\varepsilon / 2) x_{0} .
$$

This is possible because $\sigma$ is continuous at $x_{0}$. Define the functions $r(t)$ and $s(t)$ by $r(t)=0\left(0 \leq t<x_{0}-\beta\right), r(t)=\left(x_{0} \cdot \beta\right)^{-1} \cdot t-1 / \beta+1 / x_{0}\left(x_{0}-\beta \leq t<x_{0}\right), r(t)=1 / t$ $\left(x_{0} \leq t \leq 1\right), s(t)=0\left(0 \leq t<x_{0}\right), s(t)=\left(x_{0} \cdot \beta+\beta^{2}\right)^{-1} \cdot t-x_{0} \cdot\left(x_{0} \cdot \beta+\beta^{2}\right)^{-1}$ $\left(x_{0} \leq t<x_{0}+\beta\right), s(t)=1 / t\left(x_{0}+\beta \leq t \leq 1\right)$. Then $r$ and $s$ are continuous on $[0,1]$. From the Weierstrass theorem, we may choose polynomials $p(x), P(x)$ such that $0 \leq s(x)-p(x)<\varepsilon \cdot(1+2 V(1))^{-1}$ and $0 \leq P(x)-r(x)<\varepsilon \cdot(1+2 V(1))^{-1}(0 \leq x \leq 1)$. Then, evidently, $p(x) \leq s(x) \leq g(x)$ and $g(x) \leq r(x) \leq P(x)(0 \leq x \leq 1)$. Also, $\int_{0}^{1}(r(t)-g(t)) d \sigma(t)=\int_{x_{0}-\beta}^{x_{0}} r(t) d \sigma(t) \leq\left(V\left(x_{0}\right)-V\left(x_{0}-\beta\right)\right) / x_{0}<\varepsilon / 2$. But $\int_{0}^{1} P(t) d \sigma(t)<\varepsilon / 2+\int_{0}^{1} r(t) d \sigma(t)$, so

$$
\int_{0}^{1} P(t) d \sigma(t)<\varepsilon+\int_{0}^{1} g(t) d \sigma(t) .
$$

Analogously, $\int_{0}^{1}(g(t)-s(t)) d \sigma(t) \leq \int_{x_{0}}^{x_{0}+\beta}(g(t)-s(t)) d \sigma(t) \leq\left(V\left(x_{0}+\beta\right)-\right.$ $\left.V\left(x_{0}\right)\right) / x_{0}<\varepsilon / 2$. From $\int_{0}^{1} s(t) d \sigma(t)<\varepsilon / 2+\int_{0}^{1} p(t) d \sigma(t)$, we obtain

$$
\int_{0}^{1} g(t) d \sigma(t)<\varepsilon+\int_{0}^{1} p(t) d \sigma(t) .
$$

Fix $m \in\{0,1,2, \ldots\}$. Then $(1 / \varphi(x)) \cdot \sum_{n=0}^{\infty} a_{n} x^{n+m n}=\left(\varphi\left(x^{m+1}\right) / \varphi(x)\right)$. $\left(\left(\sum_{n=0}^{\infty} a_{n}\left(x^{m+1}\right)^{n}\right) / \varphi\left(x^{m+1}\right)\right)$ which tends to $c_{m}$ when $x \rightarrow 1$, by hypothesis. But $c_{m}=\int_{0}^{1} x^{m} d \sigma(x)$. Then, by linearity, $\lim _{x \rightarrow 1}(1 / \varphi(x)) \cdot \sum_{n=0}^{\infty} a_{n} x^{n} \cdot Q\left(x^{n}\right)=$ $\int_{0}^{1} Q(x) d \sigma(x)$ for all polynomials $Q(x)$. Now, since $g(x) \leq P(x)$ and $\varphi(x)$ and the $a_{n}(n=0,1,2, \ldots)$ are positive, we have $\limsup _{x \rightarrow 1}(1 / \varphi(x)) \cdot \sum_{n=0}^{\infty} a_{n} x^{n}$. $g\left(x^{n}\right) \leq \lim \sup _{x \rightarrow 1}(1 / \varphi(x)) \cdot \sum_{n=0}^{\infty} a_{n} x^{n} \cdot P\left(x^{n}\right)=\int_{0}^{1} P(t) d \sigma(t)<\int_{0}^{1} g(t) d \sigma(t)+\varepsilon$, accordingly with (2). By doing $\varepsilon \rightarrow 0$, we obtain $\lim \sup _{x \rightarrow 1}(1 / \varphi(x)) \cdot \sum_{n=0}^{\infty} a_{n} x^{n}$. $g\left(x^{n}\right) \leq \int_{0}^{1} g(t) d \sigma(t)$. If we use $p(x)$ instead of $P(x)$, it follows similarly from (3) that $\liminf _{x \rightarrow 1}(1 / \varphi(x)) \cdot \sum_{n=0}^{\infty} a_{n} x^{n} \cdot g\left(x^{n}\right) \geq \int_{0}^{1} g(t) d \sigma(t)$. Consequently, 


$$
\begin{aligned}
& \lim _{x \rightarrow 1}(1 / \varphi(x)) \cdot \sum_{n=0}^{\infty} a_{n} \cdot x^{n} \cdot g\left(x^{n}\right)=\int_{0}^{1} g(t) d \sigma(t)=\int_{x_{0}}^{1} t^{-1} d \sigma(t)=\alpha . \text { Let } \\
& x_{j}=x_{0}^{1 / j}(j=1,2, \ldots) . \text { Then } \sum_{n=0}^{\infty} a_{n} x_{j}^{n} \cdot g\left(x_{j}^{n}\right)=\sum_{n=0}^{j} a_{n}=S_{j}, \text { because } \\
& g\left(x_{j}^{n}\right)=1 / x_{j}^{n} \text { iff } x_{j}^{n} \geq x_{0} \text { iff } n \leq j \\
& =0 \text { iff } x_{j}^{n}<x_{0} \text { iff } n>j .
\end{aligned}
$$

Finally, $x_{j} \in(\delta, 1)$ for $j \geq j_{0}$ and $\lim _{j \rightarrow \infty}\left(1 / \varphi\left(x_{j}\right)\right) . \sum_{n=0}^{\infty} a_{n} x_{j}^{n} \cdot g\left(x_{j}^{n}\right)=\alpha$. Hence $\lim _{j \rightarrow \infty} S_{j} / \varphi\left(x_{0}^{1 / j}\right)=\alpha$. The theorem is proved.

It is clear that the Hardy-Littlewood theorem is a special case of this. In the classical theorem, $c_{m}=1 /(m+1)$ and $\sigma(x) \equiv x$. The point $x_{0} \in(0,1)$ is arbitrary.

We give an easy example. Let $\varphi(x)=\log \left((1-x)^{-1}\right)$. Then $\varphi\left(x_{0}^{1 / j}\right) \sim \log j(j \rightarrow$ $\infty)$ for each $x_{0} \in(0,1)$ and $c_{m}=\lim _{x \rightarrow 1}\left(\log \left(1-x^{m+1}\right)\right) / \log (1-x)=1(m=$ $0,1,2, \ldots)$. Then the sequence $\left\{c_{m}\right\}$ is completely monotonic, because it satisfies (1) trivially. The function $\sigma(x)=0(0 \leq x<1), \sigma(1)=1$ is of bounded variation, continuous at every $x_{0} \in(0,1)$ and satisfies $c_{m}=\int_{0}^{1} t^{m} d \sigma(t)(m=0,1,2, \ldots)$. In addition, $\alpha=\int_{x_{0}}^{1} 1 / t d \sigma(t)=1$. Hence, we have: If $f(x)=\sum_{n=0}^{\infty} a_{n} x^{n}(0 \leq x<1)$, $a_{n} \geq 0(n=0,1,2, \ldots)$ and $\left.\lim _{x \rightarrow 1} f(x) /\left(\log (1-x)^{-1}\right)\right)=1$, then $S_{n} \sim \log n(n \rightarrow$ $\infty)$.

REMARK. In [5, pp. 109-112], one can find several conditions which guarantee that we may choose $\sigma$ to be continuous at each $x_{0} \in(0,1)$. For the sake of completeness, we reproduce them:

(a) There exists a constant $L$ such that

$$
(1+k) \cdot\left|\lambda_{k, m}\right|<L \quad(k, m=0,1,2, \ldots ; m \leq k) .
$$

(b) There exist a number $p>1$ and a constant $L$ such that

$$
(1+k)^{p-1} \cdot \sum_{m=0}^{k}\left|\lambda_{k, m}\right|^{p}<L \quad(k=0,1,2, \ldots) .
$$

Here, $\lambda_{k, m}=\left(\begin{array}{c}k \\ m\end{array}\right)(-1)^{k-m} \cdot \Delta^{k-m} c_{m}$.

\section{REFERENCES}

1. P. Dienes, The Taylor series: An introduction to the theory of a complex variable, Dover, New York, 1957.

2. G. H. Hardy and J. E. Littlewood, Tauberian theorems concerning power series and Dirichlet's series whose coefficients are positive, Proc. London Math. Soc. (2) 11 (1911), pp. 411-478.

3. J. Karamata, Über die Hardy-Littlewoodschen Umkerhrungen des Abelschen Steligkeitssatzes, Math. Z. 32 (1930), pp. 319-320.

4. E. C. Titchmarsh, The theory of functions, 2nd ed. (corrected), Oxford Univ. Press, New York, 1968.

5. D. V. Widder, The Laplace Transform, Princeton Univ. Press, Princeton, N. J., 1946.

Departamento de AnÁlisis Matemático, Facultad de Matemáticas, C/. TARfia S. N., Sevilla 41012, Spain 